\title{
Martini, Renate
}

\section{Habilitationen und Promotionen in der Erziehungswissenschaft}

Abs, Hermann Josef [Hrsg.]; Kuper, Harm [Hrsg.]; Martini, Renate [Hrsg.]: Datenreport Erziehungswissenschaft 2020. Erstellt im Auftrag der Deutschen Gesellschaft für Erziehungswissenschaft (DGfE). Opladen; Berlin; Toronto : Verlag Barbara Budrich 2020, S. 171-186. - (Schriften der Deutschen Gesellschaft für Erziehungswissenschaft (DGfE))

Quellenangabe/ Reference:

Martini, Renate: Habilitationen und Promotionen in der Erziehungswissenschaft - In: Abs, Hermann Josef [Hrsg.]; Kuper, Harm [Hrsg.]; Martini, Renate [Hrsg.]: Datenreport Erziehungswissenschaft 2020. Erstellt im Auftrag der Deutschen Gesellschaft für Erziehungswissenschaft (DGfE). Opladen; Berlin; Toronto : Verlag Barbara Budrich 2020, S. 171-186 - URN: urn:nbn:de:0111-pedocs-189939 - DOI: 10.25656/01:18993

https://nbn-resolving.org/urn:nbn:de:0111-pedocs-189939

https://doi.org/10.25656/01:18993

in Kooperation mit / in cooperation with:

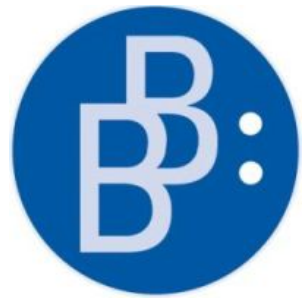

https://www.budrich.de

\section{Nutzungsbedingungen}

Dieses Dokument steht unter folgender Creative Commons-Lizenz: http://creativecommons.org/licenses/by-sa/4.0/deed.de - Sie dürfen das Werk bzw. den Inhalt vervielfältigen, verbreiten und öffentlich zugänglich machen sowie Abwandlungen und Bearbeitungen des Werkes bzw. Inhaltes anfertigen, solange sie den Namen des Autors/Rechteinhabers in der von inm festgelegten Weise nennen und die daraufhin neu entstandenen Werke bzw. Inhalte nur unter Verwendung von Lizenzbedingungen weitergeben, die mit denen dieses Lizenzvertrags identisch, vergleichbar oder kompatibel sind.

Mit der Verwendung dieses Dokuments erkennen Sie die Nutzungsbedingungen an.

\section{Terms of use}

This document is published under following Creative Commons-License: http://creativecommons.org/licenses/by-sa/4.0/deed.en - You may copy, distribute and transmit, adapt or exhibit the work or its contents in public and alter, transform, or change this work as long as you attribute the work in the manner specified by the author or licensor. New resulting works or contents must be distributed pursuant to this license or an identical or comparable license.

By using this particular document, you accept the above-stated conditions of use.

\section{Kontakt / Contact:}

\section{peDOCS}

DIPF | Leibniz-Institut für Bildungsforschung und Bildungsinformation Informationszentrum (IZ) Bildung

E-Mail: pedocs@dipf.de

Internet: www.pedocs.de

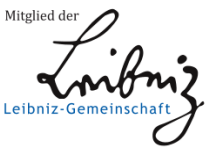




\section{Datenreport \\ Erziehungswissenschaft 2020}

Hermann Josef Abs, Harm Kuper, Renate Martini (Hrsg.)

Schriften der

Deutschen Gesellschaft für Erziehungswissenschaft

DEE Deutsche Gesellschaft 
Datenreport Erziehungswissenschaft 2020 
Schriften der Deutschen Gesellschaft für Erziehungswissenschaft (DGfE) 


\section{Hermann Josef Abs \\ Harm Kuper \\ Renate Martini (Hrsg.)}

\section{Datenreport \\ Erziehungswissenschaft 2020}

Erstellt im Auftrag

der Deutschen Gesellschaft für Erziehungswissenschaft (DGfE)

Verlag Barbara Budrich

Opladen • Berlin • Toronto 2020 


\section{Dieser Datenreport wurde aus Mitteln der Max-Traeger-Stiftung gefördert.}

Bibliografische Information der Deutschen Nationalbibliothek

Die Deutsche Nationalbibliothek verzeichnet diese Publikation in der Deutschen Nationalbibliografie; detaillierte bibliografische Daten sind im Internet über http://dnb.d-nb.de abrufbar.

(C) 2020 Dieses Werk ist bei der Verlag Barbara Budrich GmbH erschienen und steht unter der Creative Commons Lizenz Attribution-ShareAlike 4.0 International (CC BY-SA 4.0): https://creativecommons.org/licenses/by-sa/4.0/.

Diese Lizenz erlaubt die Verbreitung, Speicherung, Vervielfältigung und Bearbeitung bei Verwendung der gleichen CC-BY-SA 4.0-Lizenz und unter Angabe der UrheberInnen, Rechte, Änderungen und verwendeten Lizenz. www.budrich.de

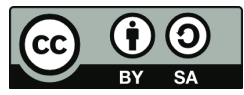

Dieses Buch steht im Open-Access-Bereich der Verlagsseite zum kostenlosen Download bereit (https://doi.org/10.3224/84742419).

Eine kostenpflichtige Druckversion (Print on Demand) kann über den Verlag bezogen werden. Die Seitenzahlen in der Druck- und Onlineversion sind identisch.

$$
\begin{array}{ll}
\text { ISBN } & 978-3-8474-2419-2 \text { (Paperback) } \\
\text { eISBN } & 978-3-8474-1551-0 \text { (PDF) } \\
\text { DOI } & 10.3224 / 84742419
\end{array}
$$

Das Werk einschließlich aller seiner Teile ist urheberrechtlich geschützt. Jede Verwertung außerhalb der engen Grenzen des Urheberrechtsgesetzes ist ohne Zustimmung des Verlages unzulässig und strafbar. Das gilt insbesondere für Vervielfältigungen, Übersetzungen, Mikroverfilmungen und die Einspeicherung und Verarbeitung in elektronischen Systemen.

Umschlaggestaltung: Bettina Lehfeldt, Kleinmachnow - www.lehfeldtgraphic.de Satz: Judith Zimmer, Hamburg - www.lektorenzimmer.de Druck: paper \& tinta, Warschau Printed in Europe 


\section{Inhalt}

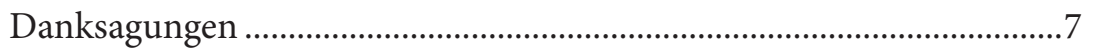

Datenreport Erziehungswissenschaft -

zwanzig Jahre indikatorenbasiertes Monitoring der Disziplin...............9

Hermann Josef Abs \& Harm Kuper

1 Studiengänge und Standorte im Hauptfach..................................... 21

Cathleen Grunert, Katja Ludwig \& Kilian Hüfner

1.1 Studiengänge und Standorte im Überblick

1.2 Studienfachbezeichnungen

1.3 Studieninhalte

1.4 Studiengangsmuster

1.5 Fazit

2 Studiengänge und Standorte der

Lehrerinnen- und Lehrerbildung .................................................... 51

Melanie Radhoff \& Christiane Ruberg

2.1 Strukturvarianten in der Lehrerinnen- und Lehrerbildung

2.2 Standorte Lehramtsstudiengänge

2.3 Bedarfsprognosen

2.4 Ausblick: Kontinuitäten und Diskontinuitäten

3 Studienabschlüsse, Übergänge und beruflicher Verbleib der Absolventinnen und Absolventen .............................................79

Christian Kerst \& Andrä Wolter

3.1 Abschlussprüfungen im Spiegel der amtlichen Statistik

3.2 Übergänge und Verbleib der Absolventinnen und Absolventen

3.3 Merkmale der Erwerbstätigkeit in mittelfristiger Perspektive

3.4 Zusammenfassung der Ergebnisse im Kontext der bisherigen Datenreporte

4 Personal

Marius Gerecht, Heinz-Hermann Krüger, Markus Sauerwein \& Johanna Schultheiß

4.1 Erziehungswissenschaftliches Personal im Innen- und Außenvergleich

4.2 Personalentwicklung im Fach Erziehungswissenschaft aus der Perspektive von Stellenausschreibungen

4.3 Geschlechterverhältnisse in den Erziehungswissenschaften

4.4 Fazit 
$5 \quad$ Forschung und Publikationskulturen ............................................... 147 Bernhard Schmidt-Hertha \& Margaretha Müller

5.1 Forschungsförderung und Drittmittel

5.2 Publikationskulturen in der Erziehungswissenschaft

5.3 Fazit

6 Habilitationen und Promotionen in der Erziehungswissenschaft.....

Renate Martini

6.1 Habilitationen und Promotionen in der Zeitschrift für Pädagogik

6.2 Promotionen und Habilitationen im Fächervergleich

6.3 Fazit

7 Der Stellenmarkt von wissenschaftlichem Nachwuchs in Erziehungswissenschaft/Bildungsforschung

Annette Stelter

7.1 Methoden

7.2 Umfang und Inhalt der Stellenausschreibungen

7.3 Weiterqualifikationsmöglichkeiten für wissenschaftlichen Nachwuchs

7.4 Zusammenfassung und Fazit der Analyse der Stellenausschreibungen

Tabellenanhang. 197

Die Autorinnen und Autoren 


\section{Habilitationen und Promotionen in der Erziehungswissenschaft}

Renate Martini ${ }^{1}$

Abgeschlossene Habilitationen und Promotionen sind wichtige Indikatoren wie auch eine wesentliche Grundlage für die Entwicklung einer Wissenschaftsdisziplin. Zugleich bilden sie eine Basis für die kontinuierliche Qualitätssicherung in der Forschung. Daher untersucht dieses Kapitel die Habilitationen und Promotionen separat von anderen Indikatoren zur Beschreibung von Forschungsstärke einer sozialwissenschaftlichen Disziplin oder Einrichtung. Gleichwohl steht der Indikator Abschluss von Habilitationen und Promotionen in Wechselbeziehung zu anderen Indikatoren wie Personalsituation (vgl. Kapitel 4) und Drittmittelakquise (vgl. Kapitel 5).

Erziehungswissenschaftliche Institute und Fachbereiche werden jährlich gebeten, die an ihren Einrichtungen abgeschlossenen Habilitationen und Promotionen an die Zeitschrift für Pädagogik (ZfPäd) zu melden. Mit zahlreichen Informationen (u.a. Thema, Einrichtung, Sprache) lassen sie differenziertere Analysen zu, als es bei Angaben aus statistischen Ämtern möglich wäre. Daher steht zunächst die Auswertung dieses Datenkorpus im Vordergrund. Anschließend wird der Frage nachgegangen, wie sich die Erziehungswissenschaft im Vergleich zu den Fächern Psychologie, Politikwissenschaften, Sozialwissenschaften sowie Wirtschaftswissenschaften entwickelt. Diese Gegenüberstellung wird auf der Basis der Hochschulstatistik des Statistischen Bundesamts ${ }^{2}$ vorgenommen.

Weil dieser Datenreport kein eigenes genderspezifisches Kapitel beinhaltet, wird hier der Anteil von Frauen und Männern bei den einzelnen Aspekten besonders berücksichtigt.

Zum zwanzigjährigen Bestehen des Datenreports Erziehungswissenschaft kann auf eine große Zeitreihe zurückgegriffen werden, die Entwicklungen und Trends noch deutlicher hervortreten lässt als zeitlich segmentierte Abschnitte. Eine jährliche Darstellung seit dem Jahr 2000 lässt sich allein aufgrund des Umfangs nicht realisieren, dennoch zeigen auch die beispielhaft ausgewählten Zeitpunkte Veränderungen auf.

1 Für wichtige Hinweise danke ich Horst Weishaupt, Hermann Avenarius sowie Johannes Hiebl.

2 In Anlehnung an die Bezeichnung der Fächergruppen beim Statistischen Bundesamt. Unter Sozialwissenschaften werden dort Sozialkunde, Sozialwissenschaft und Soziologie zusammengefasst. 


\subsection{Habilitationen und Promotionen in der Zeitschrift für Pädagogik}

Mit der Meldung von Habilitationen und Promotionen aus erziehungswissenschaftlichen Instituten und Fachbereichen an die Zeitschrift für Pädagogik (ZfPäd) steht ein Datenkorpus zur Verfügung, das neben der Häufigkeit der jährlichen Abschlüsse und der Geschlechterverteilung auch Aussagen zu Sprache, inhaltlicher Schwerpunktsetzung wie auch der Entwicklung an einzelnen Hochschulen zulässt. Das wird nachfolgend konkretisiert.

\subsubsection{Analysebasis und Methodik}

Im Anschluss an den letzten Datenreport, der die Habilitationen und Promotionen aus den Jahren 2009 bis 2014 in den Blick genommen hatte, stellen diese Analysen die in den Jahren 2015 bis 2018 an deutschen Hochschulen abgeschlossenen Arbeiten in den Vordergrund. Für die Darstellung von Entwicklungen werden auch die Meldungen für die Jahre 1995, 2000, 2005 sowie 2010 in die Abbildungen einbezogen. Um Trends noch besser erkennen zu können, fließen auch die Werte für den vergleichbaren Zeitraum 2011 bis 2014 bzw. ab 2000 durchgängig in die Diskussion ein.

Bei der Interpretation der Zahlen sind u. a. folgende Aspekte in Betracht zu ziehen (siehe auch Kauder 2012: 735ff.):

a) Nichtberücksichtigung von Qualifizierungsarbeiten: Fachdidaktische Habilitationen und Promotionen werden ebenso wie Arbeiten in der Pädagogischen Psychologie häufig in der Fachdisziplin angefertigt und von Prüfungskommissionen eben dieser Disziplinen abgenommen, sodass sie den erziehungswissenschaftlichen Fakultäten und Instituten nicht bekannt sind. Daraus folgt, dass sie nur partiell, in Abhängigkeit von der Hochschulstruktur in die Statistiken der ZfPäd einfließen.

b) Datenschutz: Alljährlich begründen immer wieder neue Einrichtungen den bewussten Verzicht auf Meldungen an die Zeitschrift für Pädagogik mit datenschutzrechtlichen Bedenken. Bei der Angabe von Habilitand/Habilitandin, Doktorand/Doktorandin, bearbeitetem Thema sowie Gutachterinnen und Gutachtern, wie es in der ZfPäd erfolgt, handelt es sich allerdings nicht um Daten, die einem besonderen Schutz unterliegen. Im Übrigen sind derartige Angaben über Bibliothekskataloge und Fachdatenbanken allgemein zugänglich. Zudem ist die Pflicht zu publizieren bei Dissertationen ja von Rechts wegen geregelt.

c) Korrektur von Angaben: Sind Arbeiten durch Standortwechsel der Betreuer innerhalb eines Jahres mehrfach gemeldet worden oder wurden Arbeiten in aufeinanderfolgenden Jahren wiederholt genannt, werden auf der Basis 
der Angaben in der Deutschen Nationalbibliothek oder auch persönlicher Nachfragen entsprechende Korrekturen vorgenommen. Daher sind die hier verwendeten Werte nicht identisch mit Zählungen innerhalb der ZfPäd. Nachmeldungen werden dem gemeldeten Abschlussjahr zugeordnet.

d) Inhaltsanalyse: Die Inhaltsanalyse für die Habilitationen und Promotionen der Jahre 2009 bis 2014 wurde auf der Basis der in der »FIS Bildung Literaturdatenbank « vorhandenen Verschlagwortung und deren fachsystematischer Zuordnung (vgl. Martini/Schmidt-Hertha 2012: 188ff.) vorgenommen. Da bereits im letzten Datenreport die Treffsicherheit bei der Zuordnung mit dieser Methode punktuell nicht gegeben war, werden die Arbeiten für diesen Datenreport individuell einem inhaltlichen Schwerpunkt, bezeichnet mit Forschungsfeld, zugeordnet. Grundlage für die Entscheidung sind der Titel, die Schlagwörter, das Abstract und ggf. das Inhaltsverzeichnis der einzelnen Arbeit. Die Sektionen der DGfE bilden die Basis für die inhaltliche Orientierung bei der Klassifizierung. Arbeiten, die mehrere Forschungsfelder thematisieren oder nicht eindeutig zugeordnet werden können, werden der Rubrik »Mehrfachzuordnung« zugeführt. Um Entwicklungstendenzen über einen längeren Zeitraum erkennen zu können, werden in diese Clusterung auch die gemeldeten Habilitationen und Promotionen für die Jahre 1995, 2000, 2005 und 2010 einbezogen.

\subsubsection{Erziehungswissenschaftliche Habilitationen}

Die für den Untersuchungszeitraum 2015 bis 2018 an die Zeitschrift für Pädagogik gemeldeten Habilitationen schwanken zwischen 24 und 30 Arbeiten jährlich. Die 107 Habilitationen, die zwischen 2015 und 2018 an deutschen Hochschulen zum Abschluss kamen, werden von 46 Hochschuleinrichtungen an die ZfPäd gemeldet. Das sind acht Habilitationen mehr als im Vergleichszeitraum 2011-2014. An nur vier Universitäten kommen über diesen Zeitraum zwischen fünf und acht Verfahren zum Abschluss. Alle anderen Hochschulen liegen darunter.

Insgesamt verstärkt sich der Trend, dass sich nach der Einführung der Juniorprofessur 2002 die Zahl der Habilitationen etwa um ein Drittel reduziert. Die Daten aus dem Statistischen Bundesamt bestätigen diese Entwicklung. Allerdings zeigt sich nach den beiden deutlichen Rückgängen in den Jahren 2010 und 2013 ein Ansteigen auf eine durchschnittliche Häufigkeit von 26 Habilitationen. Auch wenn die Zahl der Juniorprofessuren in den letzten Jahren auf 89 angewachsen ist (vgl. Kapitel 4), kann ihre Karriereentwicklung noch nicht hinreichend eingeschätzt werden. ${ }^{3}$ Für die Erziehungswissenschaft wird näher

3 Auch umfangreiche Darstellungen zur Situation des wissenschaftlichen Nachwuchses wie etwa der Bundesbericht Wissenschaftlicher Nachwuchs (BuWiN) geben keine Auskunft darüber. Die Daten zur Erziehungswissenschaft werden dort der Fächergruppe Rechts-, Wirtschafts- und Sozialwissenschaften zugeordnet und lassen somit keine Spezifizierung zu. Auch die Analysen der Deutschen Gesellschaft Juniorprofessur nutzen diese Fächergruppe, sodass auch hier keine Informationen zur Situation in der Erziehungswissenschaft gegeben werden. 
zu untersuchen sein, ob die Juniorprofessur die Habilitation dauerhaft ersetzen oder als eine von mehreren möglichen Alternativen neben der Habilitation verstanden wird. Die unterschiedliche Besoldungshöhe der Juniorprofessuren in den einzelnen Bundesländern wie auch zumeist nicht ruhegehaltsfähige Leistungsbezüge für besondere Leistungen könnten die Entscheidung für diesen Karriereweg beeinflussen (vgl. Preißler 2019).

Abbildung 6.1: Erziehungswissenschaftliche Habilitationen und Anteile von Frauen und Männern 1995, 2000, 2005 und 2010-2018

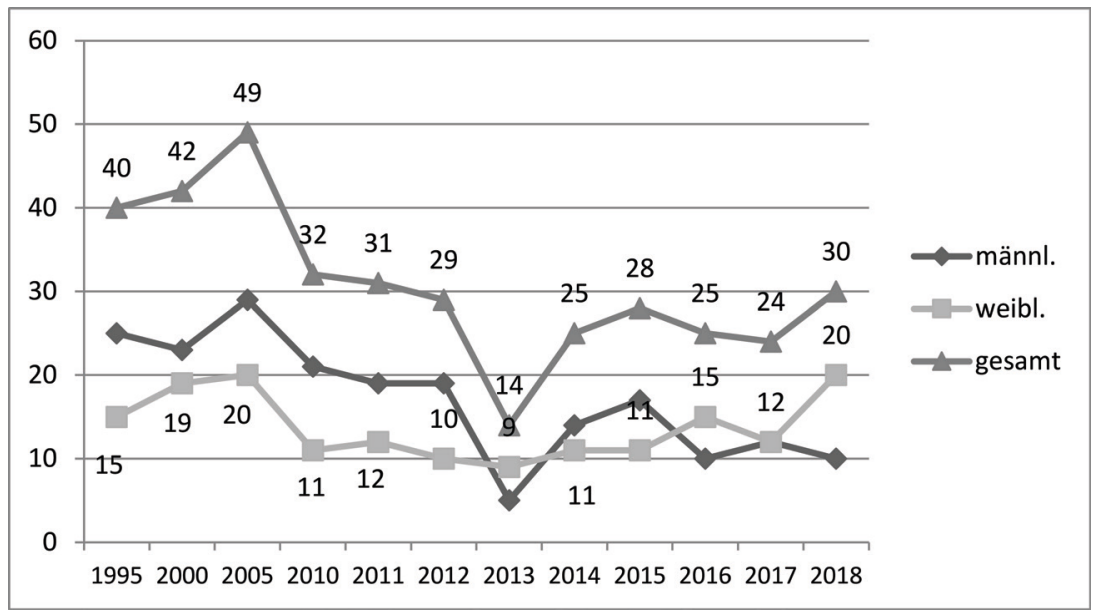

Quelle: Zeitschrift für Pädagogik; eigene Berechnungen

Der Anteil der von Frauen zwischen 2015 und 2018 abgeschlossenen Habilitationsverfahren liegt zwischen $40 \%$ und $60 \%$ und schwankt von Jahr zu Jahr. Bei den 107 abgeschlossenen Habilitationsverfahren sind die Ergebnisse von 5 Arbeiten in englischer Sprache publiziert, 4 davon wurden von Frauen geschrieben. Im Untersuchungszeitraum sind 46 (zuvor 18) kumulative Habilitationen entstanden, die mit 23 (zuvor 8) Frauen und 23 (zuvor 10) Männern nach wie vor nicht auf ein geschlechtsspezifisches Interesse bei der Auswahl dieser Abschlussform deuten.

Hinsichtlich der inhaltlichen Schwerpunktsetzung werden bei den Habilitationen überwiegend Themen aus den Forschungsfeldern Bildungsforschung, Lernen, Fachdidaktik und Bildungstheorie aufgegriffen. Aufgrund der kleinen Fallzahlen lassen sich keine Trends erkennen. Allerdings zeigen die Daten für die Bildungsforschung seit dem Jahr 2000 einen Anstieg bis 2005 auf 15 Arbeiten, dem dann ein steter Rückgang bis auf 4 Habilitationen in 2018 folgt. Für ein Fünftel der Habilitationen konnte aufgrund einer thematischen Vielfalt keine eindeutige Zuordnung vorgenommen werden. In 
den zehn identifizierten Forschungsfeldern ist in fünf dieser Themenfelder der Anteil der Frauen größer als der der Männer.

Tabelle 6.1: Verteilung der Habilitationen nach Forschungsfeldern 2015-2018 mit Anteil der Frauen

\begin{tabular}{lcccc} 
& \multicolumn{2}{c}{ Habilitationen } & \multicolumn{2}{c}{ Frauen } \\
Forschungsfeld & absolut & in $\%$ & absolut & in $\%$ \\
Berufs- U. Wirtschaftspädagogik & 7 & 6,5 & 4 & 57,1 \\
\hline Berufsbildung & 0 & 0 & 0 & 0 \\
\hline Bildungsforschung & 17 & 15,9 & 10 & 58,8 \\
\hline Bildungspolitik & 1 & 0,9 & 0 & 0 \\
\hline Bildungstheorie & 11 & 10,3 & 5 & 45,4 \\
\hline Fachdidaktik & 12 & 11,2 & 6 & 50,0 \\
\hline Frühe Bildung & 3 & 2,8 & 2 & 66,7 \\
\hline Hochschule/Studium & 1 & 0,9 & 1 & 100,0 \\
\hline Lehrerbildung & 6 & 5,6 & 3 & 50,0 \\
\hline Lernen/Päd. Psychologie & 16 & 14,9 & 11 & 68,7 \\
\hline Schulsystem/Schule & 0 & 0 & 0 & 0 \\
\hline Sonderpädagogik & 4 & 3,7 & 13 & 25,0 \\
\hline Sozialpädagogik & 21 & 7,5 & 25,0 \\
\hline Mehrfachzuordnung & 8 & 62,0
\end{tabular}

Quelle: Zeitschrift für Pädagogik; eigene Zuordnungen

\subsubsection{Erziehungswissenschaftliche Promotionen}

Für den Zeitraum 2015 bis 2018 werden insgesamt 1.727 Promotionen von deutschen Hochschulen an die Zeitschrift für Pädagogik gemeldet, das sind 10 Arbeiten weniger als im vergleichbaren Untersuchungszeitraum 2011-2014 (vgl. Martini 2016: 183f). Seit 2016 hat sich die Zahl der Promotionen allerdings um insgesamt 83 Arbeiten verringert. Im Untersuchungszeitraum haben 1.107 Frauen und 585 Männer ihr Promotionsverfahren abgeschlossen. Für 35 Personen war das Geschlecht nicht zu ermitteln.

Offensichtlich gibt es bei den Promotionen in der Erziehungswissenschaft im Rhythmus von etwa vier Jahren eine Wellenbewegung. Das wird insbesondere ab 2010 deutlich, vgl. Abbildung 6.2. Nach einem Rückgang, der zumeist über zwei Jahre verläuft, ist nach dem Tiefstwert in den beiden Folgejahren ein zumindest ebenso großer Zuwachs zu verzeichnen. Rückgang und Anstieg kompensieren sich nahezu. 
Da bei der Personalstärke der Professuren solche Schwankungen ausbleiben, besteht eine mögliche Erklärung darin, dass die Kapazität der betreuenden Professuren ausgeschöpft ist und erst nach Abschluss von Promotionsverfahren neue Betreuungsverhältnisse eingegangen werden können.

Abbildung 6.2: Erziehungswissenschaftliche Promotionen in der ZfPäd und Anteile von Frauen und Männern 1995, 2000, 2005 und 2010-2018

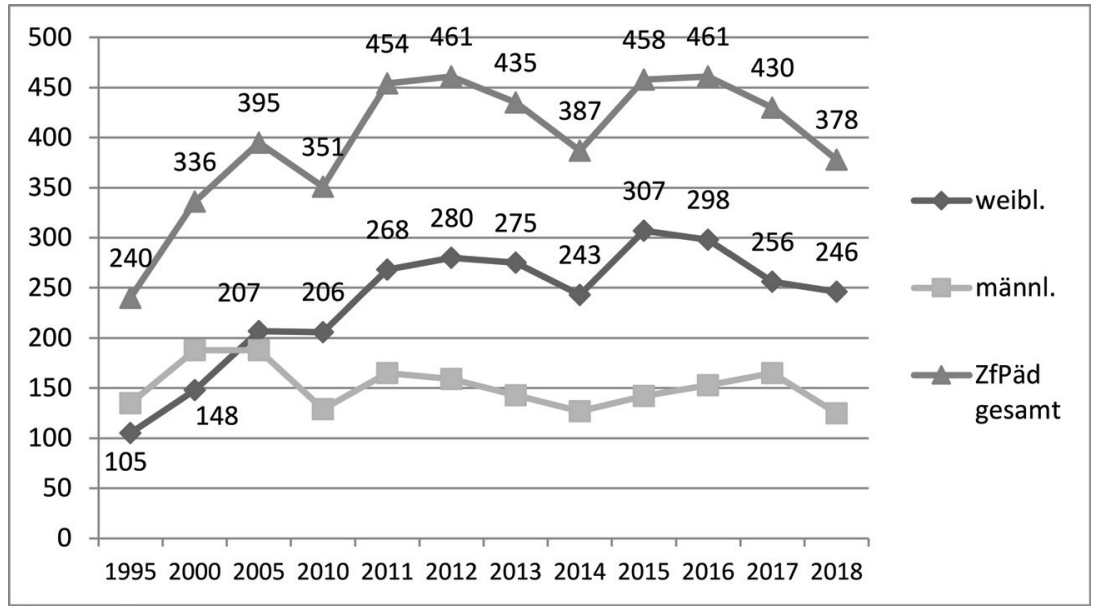

Quelle: Zeitschrift für Pädagogik; eigene Berechnungen

Promovieren bis zum Jahr 2000 mehr Männer als Frauen, so ist nach dem Gleichstand in 2005 ein deutlicher Anstieg des Frauenanteils zu verzeichnen. Ab 2012 schließen nahezu doppelt so viele Frauen wie Männer diese Qualifikationsform ab. Im Jahr 2017 verringert sich dieser Abstand etwas. Im Jahr 2015 erreicht der Anteil 67 Prozentpunkte. In den Folgejahren stabilisiert er sich bei rund $63 \%$. Damit liegt der Frauenanteil bei den Promotionen in der Erziehungswissenschaft 20 Prozentpunkte über dem mittleren Wert aller beim Statistischen Bundesamt dokumentierten Disziplinen.

Die im Zeitraum 2015 bis 2018 abgeschlossenen Promotionen werden von 77 deutschen Hochschulen gemeldet. An 14 Einrichtungen werden mehr als 40 Verfahren in diesem Zeitraum zum Abschluss geführt. Beim letzten Datenreport waren es noch 23 Hochschulen, die im vergleichbaren Zeitraum mehr als 40 Arbeiten abschließen konnten. Nur von der Universität Hamburg werden in dem jetzt untersuchten Zeitraum mehr als 100 Promotionsverfahren gemeldet. Für den Zeitraum 2011 bis 2014 wurden noch fünf Hochschulen mit dieser Häufigkeit genannt.

Über den Analysezeitraum hinweg werden zum Teil erhebliche Veränderungen innerhalb einer Einrichtung deutlich. An 11 Hochschulen ist innerhalb 
der letzten vier Jahre ein Rückgang der Promotionen im Vergleich zu den Jahren 2011 bis 2014 um mehr als 10 Arbeiten zu verzeichnen. An den Universitäten in Bamberg, Erlangen-Nürnberg, Dortmund, Lüneburg, Köln sowie der Freien Universität Berlin hat sich die Anzahl der Promotionen sogar um mehr als 15 verringert. 11 Hochschulen haben in den letzten Jahren aber auch einen Aufwuchs von mehr als 10 Arbeiten erlangen können. In Ludwigsburg fällt dieser Aufwuchs mit 23 Promotionen, in Hamburg mit 29 und in Hildesheim mit 39 Arbeiten besonders deutlich aus.

Für fremdsprachige Publikationen ist bei Promotionen seit dem Jahr 2000 ein sehr verhaltener Anstieg zu beobachten (vgl. Martini/Dees 2012: 48). Für den Untersuchungszeitraum kann ein leichtes, aber kontinuierliches Wachstum auf etwa $9 \%$ konstatiert werden, was immerhin eine Verdopplung seit 2009 bedeutet. Die Freie Universität Berlin (18), die LMU (16) und die Technische Universität (9) - beide in München - sowie die Universität in Oldenburg (8) haben besonders zu dieser Entwicklung beigetragen. In allen Jahren publizieren fast doppelt so viele Frauen wie Männer ihre Untersuchungsergebnisse in englischer Sprache.

Tabelle 6.2: Verteilung der Promotionen nach Forschungsfeldern 2015-2018 mit Anteil der Frauen

\begin{tabular}{lcccc} 
& \multicolumn{2}{c}{ Promotionen } & \multicolumn{2}{c}{ Frauen } \\
Forschungsfeld & absolut & in $\%$ & absolut & in \% \\
Berufs- u. Wirtschaftspädagogik & 115 & 6,6 & 73 & 63,5 \\
\hline Berufsbildung & 42 & 2,4 & 23 & 54,7 \\
\hline Bildungsforschung & 338 & 19,6 & 206 & 60,9 \\
\hline Bildungspolitik & 21 & 1,2 & 10 & 47,6 \\
\hline Bildungstheorie & 58 & 3,4 & 33 & 56,9 \\
\hline Fachdidaktik & 141 & 8,2 & 83 & 58,9 \\
\hline Frühe Bildung & 68 & 3,9 & 54 & 79,4 \\
\hline Hochschule/Studium & 61 & 3,5 & 43 & 70,5 \\
\hline Lehrerbildung & 143 & 8,3 & 95 & 66,4 \\
\hline Lernen/Päd. Psychologie & 182 & 10,3 & 135 & 74,2 \\
\hline Schulsystem/Schule & 44 & 2,5 & 115 & 61,4 \\
\hline Sonderpädagogik & 67 & 3,9 & 169 & 62,7 \\
\hline Sozialpädagogik & 262 & 15,7 & 64,5 \\
\hline Mehrfachzuordnung & 185 & 42 & 27 \\
\hline
\end{tabular}

Quelle: Zeitschrift für Pädagogik; eigene Zuordnungen 
Das kumulative Verfahren zur Publikation der Forschungsergebnisse aus einem Promotionsverfahren hat auch in der Erziehungswissenschaft Fuß gefasst (vgl. auch DGfE 2011). Die ZfPäd hat 2014 dieses Charakteristikum in die Befragung der Hochschulen aufgenommen; es wurden damals 16 publikationsbasierte Promotionen gemeldet. Diese Zahl hat sich 2015 verdoppelt, nahm dann geringfügig ab und ist im Jahr 2018 auf 47 Arbeiten angewachsen. Sie hat sich damit in den letzten vier Jahren fast verdreifacht und macht 7,8 \% aller erziehungswissenschaftlichen Promotionen aus. Die Hochschulen in Dortmund (11), Kiel (18), Ludwigsburg (13), Oldenburg (14), Tübingen (14) sowie die Freie Universität Berlin (10) stützen diese Entwicklung besonders. Auch hier ist der Anteil der Frauen zumeist doppelt so groß wie der der Männer. Berücksichtigt man allerdings die Relation zwischen Frauen und Männern am Gesamtkorpus, dann haben im Abschlussjahr 2017, und nur dann, sowohl bei der Nutzung der englischen Sprache als auch beim kumulativen Abschlussverfahren die Männer den größeren Anteil.

Zur Ermittlung der inhaltlichen Schwerpunkte erziehungswissenschaftlicher Promotionen werden die 1.727 von 2015 bis 2018 an die ZfPäd von deutschen Hochschulen gemeldeten Promotionen ausgewählten Forschungsfeldern zugeordnet. Die Verteilung auf die einzelnen Forschungsfelder sowie der Anteil von Frauen an dem thematischen Schwerpunkt werden in Tabelle 6.2 dargestellt.

Für die Forschungsfelder Bildungsforschung, Sozialpädagogik, Lernen, Fachdidaktik, Lehrerbildung sowie Berufs- und Wirtschaftspädagogik werden die meisten Promotionen gemeldet, wobei sich bei der Lehrerbildung seit 2010 der größte Anstieg zeigt. Das dürfte insbesondere auf die speziellen Förderaktivitäten im BMBF-Rahmenprogramm zur Förderung der empirischen Bildungsforschung sowie die Qualitätsoffensive Lehrerbildung zurückzuführen sein.

In der Bildungsforschung, dem Forschungsfeld mit der größten thematischen Heterogenität lassen sich einige Veränderungen erkennen. Die Bildungsgeschichte macht innerhalb der Bildungsforschung mit $26,7 \%$ zwar noch immer den größten Anteil aus, die Anzahl der abgeschlossenen Promotionen hat sich seit dem Jahr 2000 aber auf ein Drittel reduziert. Fast genauso häufig wie die Bildungsgeschichte werden bildungssoziologische Themen gewählt. Mit nur etwa halb so großer Häufigkeit werden Themen der Biografieforschung, Medienpädagogik, Genderforschung sowie der Wissenschaftsforschung untersucht. Alle anderen Teilbereiche der Bildungsforschung wie etwa die Freizeit- oder Umweltpädagogik werden deutlich seltener in einem Dissertationsthema bearbeitet. 263 Arbeiten lassen sich aufgrund ihrer heterogenen Thematik nicht eindeutig zuordnen.

Obwohl insgesamt deutlich mehr Frauen als Männer promovieren, zeigen deren Anteile innerhalb einzelner Teildisziplinen ein unterschiedliches Bild. In den Themenfeldern Berufsbildung, Bildungspolitik, Bildungstheorie und Fachdidaktik zeigt sich ein annähernd ausgewogenes Verhältnis der Geschlechter. 
Wohingegen in den Forschungsfeldern frühkindliche Bildung, Hochschule/ Studium sowie Lernen/Pädagogische Psychologie mit einem Anteil von nahezu zwei Drittel eine deutlich stärkere Repräsentanz der Frauen vorliegt. Eine »Männerdomäne« bei der Themenwahl lässt sich nicht identifizieren.

Da im nächsten Abschnitt die Daten des Statistischen Bundesamts herangezogen werden, muss an dieser Stelle auf die Unterschiede zwischen beiden Datenquellen eingegangen werden. Auf der Basis des Hochschulstatistikgesetzes bekommt das Statistische Bundesamt die Daten für die Promovierendenstatistik über die Statistischen Landesämter von den Prüfungsämtern der jeweiligen Hochschulen gemeldet (siehe auch Vollmar 2019). Die Daten werden zum 1. Dezember eines jeden Jahres auf der Basis der abgelegten Prüfungen (Disputation) erhoben. Die Redaktion der Zeitschrift für Pädagogik schreibt die erziehungswissenschaftlichen Fachbereiche und Institute der Hochschulen in Deutschland, Österreich und der Schweiz Mitte Januar an und bittet um die Meldung der im vorherigen Kalenderjahr abgeschlossenen Habilitationen und Promotionen. Die Beteiligung aus den einzelnen Hochschulen - wir fokussieren hier Deutschland - ist überaus heterogen. Zahlreiche Hochschulen übermitteln die erbetenen Informationen zügig und im gewünschten Format. Andere Einrichtungen müssen mehrfach erinnert werden, und selbst das ist nicht stets mit einer Rückmeldung verbunden. Daher sind Abweichungen zwischen den Daten des Statistischen Bundesamts und der Zeitschrift für Pädagogik zu erwarten.

Abbildung 6.3: Jährlich ${ }^{1}$ gemeldete Promotionen an die ZfPäd und Daten des Statistischen Bundesamts 1995, 2000-2018

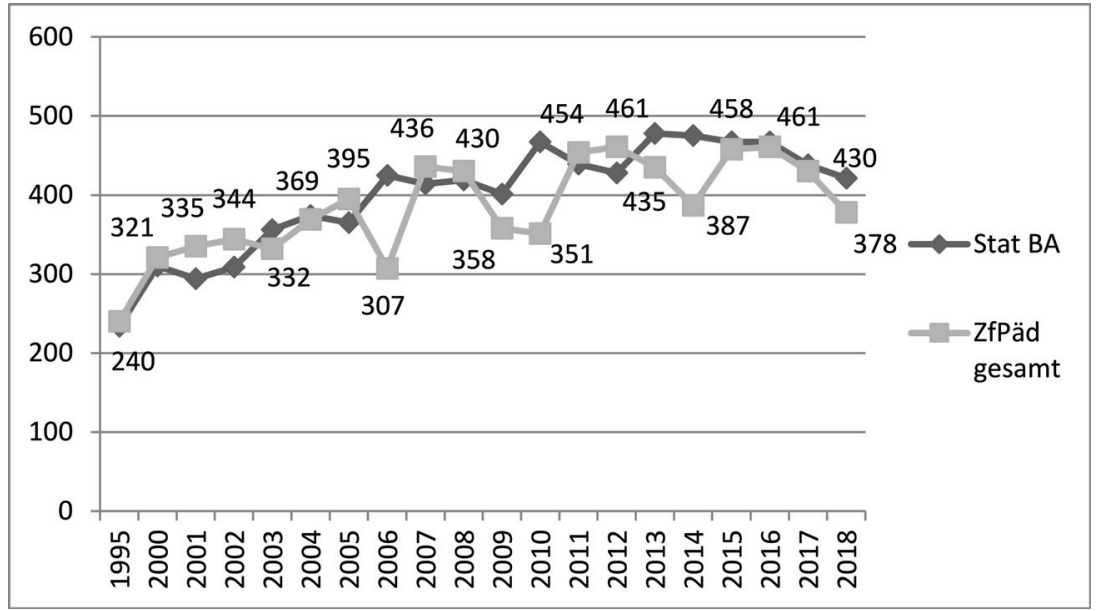

1 Die Zahlen repräsentieren nur die Meldungen an die Zeitschrift für Pädagogik.

Quelle: Statistisches Bundesamt, Fachserie 11, Reihe 4.2; Zeitschrift für Pädagogik 
In der ZfPäd ist seit 2000 - ähnlich dem Statistischen Bundesamt - zunächst ein Anwachsen der Promotionen bis 2005 zu verzeichnen, vgl. Abbildung 6.3. Danach setzen kräftige Schwankungen ein, die sich z.B. nicht durch Veränderungen in der redaktionellen Zuständigkeit seitens der Zeitschrift begründen lassen. Auch nehmen die Abweichungen zu den Daten des Statistischen Bundesamts deutlich zu. In den Jahren 2006 und 2010 beträgt der Unterschied jeweils mehr als 100 Promotionen, 2014 fast 90 Arbeiten. Die Graphen vermitteln den Eindruck, als würden die Daten die ZfPäd mit einer Verzögerung erreichen. In der ersten Dekade dieses Jahrhunderts liegt diese Verschiebung bei einem, später bei zwei Jahren. Erst in den letzten Jahren gelingt eine aktuelle Datenübermittlung.

Ursache dafür können hochschulinterne Prozesse sein. Allerdings dürfte auch die unterschiedliche Meldepraxis an die ZfPäd nicht ohne Einfluss bleiben. Die eine Hochschule übermittelt die Angaben nach Abschluss der Disputation, eine andere erst bei Vorliegen der Publikation, manche informieren sogar zu beiden Zeitpunkten. Auch wenn das jährliche »Einsammeln« der Habilitationen und Promotionen mit erheblichem Aufwand verbunden ist, so scheint eine stärkere Abstimmung mit den Hochschulen und ggf. eine Erinnerung an vorhandene Absprachen (Meldung nach der Disputation) zur Verbesserung der Datenlage wünschenswert.

\subsection{Promotionen und Habilitationen im Fächervergleich}

Dieser Abschnitt geht der Frage nach, wie sich die Erziehungswissenschaft im Vergleich zu anderen Fachdisziplinen entwickelt hat. Für den Vergleich der Promotionen und Habilitationen aus der Erziehungswissenschaft werden - wie in bisherigen Datenreporten - die Fachdisziplinen Psychologie, Politikwissenschaften, Sozialwissenschaften sowie Wirtschaftswissenschaften herangezogen. Die Datengrundlage bildet die Hochschulstatistik des Statistischen Bundesamts. Nur sie ermöglicht einen fachdisziplinären Bezug, wenngleich nur zur Gesamtzahl der abgeschlossenen Arbeiten sowie zum Geschlechteranteil.

\subsubsection{Promotionen im Fächervergleich}

Aus der Hochschulstatistik des Statistischen Bundesamts (vgl. Tabelle 6.3) wird ersichtlich, dass sich in allen Fachgebieten die Häufigkeit von Promotionen in den Jahren seit 1995 deutlich erhöht hat. Bei den Steigerungsraten werden aber Unterschiede deutlich. In der Psychologie (163\%) und den Sozialwissenschaften (114\%) konnte die Anzahl mehr als verdoppelt werden. In 
der Erziehungswissenschaft wurde eine Steigerung um $80 \%$ erreicht. In den Politik- und Wirtschaftswissenschaften liegt das Wachstum bei 43 \%. Die Erziehungswissenschaft liegt damit im Mittelfeld, allerdings deutlich unter dem Niveau des Anstiegs in der Psychologie. Bedauerlich ist, dass das Fachgebiet Psychologie beim Statistischen Bundesamt auch nach der Neukonzipierung der Fächergruppen keinerlei Untergliederung erfährt, sodass die für die Erziehungswissenschaft interessanten Arbeiten aus der Pädagogischen Psychologie weder kenntlich gemacht noch wahrgenommen werden können. Seit 2016 ist die Anzahl abgeschlossener Promotionen in allen Fachgebieten um 10 bis 15 Prozentpunkte rückläufig.

Tabelle 6.3: Abgeschlossene Promotionen nach Fachgebieten 1995, 2000, 2005, 2010 und 2015-2018

\begin{tabular}{|c|c|c|c|c|c|}
\hline & $\begin{array}{l}\text { Erziehungs- } \\
\text { wissenschaft }^{1}\end{array}$ & Psychologie & $\begin{array}{l}\text { Politik- } \\
\text { wissenschaft }\end{array}$ & $\begin{array}{l}\text { Sozial- } \\
\text { wissenschaft }\end{array}$ & $\begin{array}{l}\text { Wirtschafts- } \\
\text { wissenschaft }\end{array}$ \\
\hline 1995 & 243 & 223 & 188 & 181 & 924 \\
\hline 2000 & 310 & 301 & 262 & 238 & 1.024 \\
\hline 2005 & 365 & 486 & 284 & 278 & 1.208 \\
\hline 2010 & 467 & 509 & 274 & 262 & 1.226 \\
\hline 2015 & 467 & 544 & 303 & 354 & 1.037 \\
\hline 2016 & 467 & 598 & 305 & 375 & 1.450 \\
\hline 2017 & 438 & 589 & 273 & 388 & 1.303 \\
\hline 2018 & 421 & 533 & 257 & 328 & 1.284 \\
\hline \multicolumn{6}{|c|}{ Promotionen je 100 Professuren } \\
\hline 1995 & 22,2 & 42,2 & 68,9 & 35,1 & 69,3 \\
\hline 2000 & 31,6 & 57,4 & 96,7 & 50,1 & 75,5 \\
\hline 2005 & 42,3 & 83,9 & 91,6 & 63,8 & 86,2 \\
\hline 2010 & 50,0 & 88,2 & 86,4 & 57,1 & 72,2 \\
\hline 2015 & 46,5 & 83,0 & 88,1 & 70,4 & 49,9 \\
\hline 2016 & 45,6 & 87,0 & 87,9 & 75,6 & 69,5 \\
\hline 2017 & 42,5 & 84,9 & 76,9 & 76,2 & 60,1 \\
\hline 2018 & 40,3 & 74,6 & 69,5 & 62,8 & 58,5 \\
\hline
\end{tabular}

1 einschließlich Sonderpädagogik und Sozialwesen

Quelle: Statistisches Bundesamt, Fachserie 11, Reihe 4.2 und 4.4; eigene Berechnungen

Seit 2010 wächst die Zahl der Professuren in der Erziehungswissenschaft um insgesamt 23 \%, ähnlich der Entwicklung in der Psychologie (21 \%). Das wirkt sich allerdings nicht auf die Zahl der Promotionen je 100 Professuren aus. Wie Tabelle 6.3 verdeutlicht, liegt die Zahl der Promotionen je Professur weiterhin 
deutlich unter den zum Vergleich herangezogenen Disziplinen. In der Erziehungswissenschaft wird nur alle zwei Jahre ein Promotionsverfahren je Professur abgeschlossenen, bei rückläufiger Tendenz.

Diese Entwicklung verwundert angesichts diverser Förderprogramme zur Unterstützung des wissenschaftlichen Nachwuchses, die seit 2008 eingerichtet wurden. Allerdings lässt die Zunahme der Drittmittel in der Erziehungswissenschaft um 60 \% seit 2010 - im Vergleich zur Psychologie (38 \%), Politik- (34 \%), Sozial- (43\%) und Wirtschaftswissenschaften (26\%) - auch vermuten, dass zahlreiche personelle Kapazitäten an die Einwerbung von Drittmitteln gebunden werden (vgl. Kapitel 5.1). Damit würde dem Indikator Drittmittelakquise eine höhere Priorität als dem der Förderung des wissenschaftlichen Nachwuchses eingeräumt. Weitere Gründe für diese Diskrepanz können aber auch in einer stärkeren Anwendungsorientierung geförderter Projekte, der Zunahme befristeter Stellen (vgl. Kapitel 4.1) oder stärkeren personellen Veränderungen innerhalb einer Hochschule liegen.

Abbildung 6.4: Prozentualer Anteil von Frauen bei Promotionen nach Fachgebiet 1995, 2000, 2005, 2010 und 2015-2018

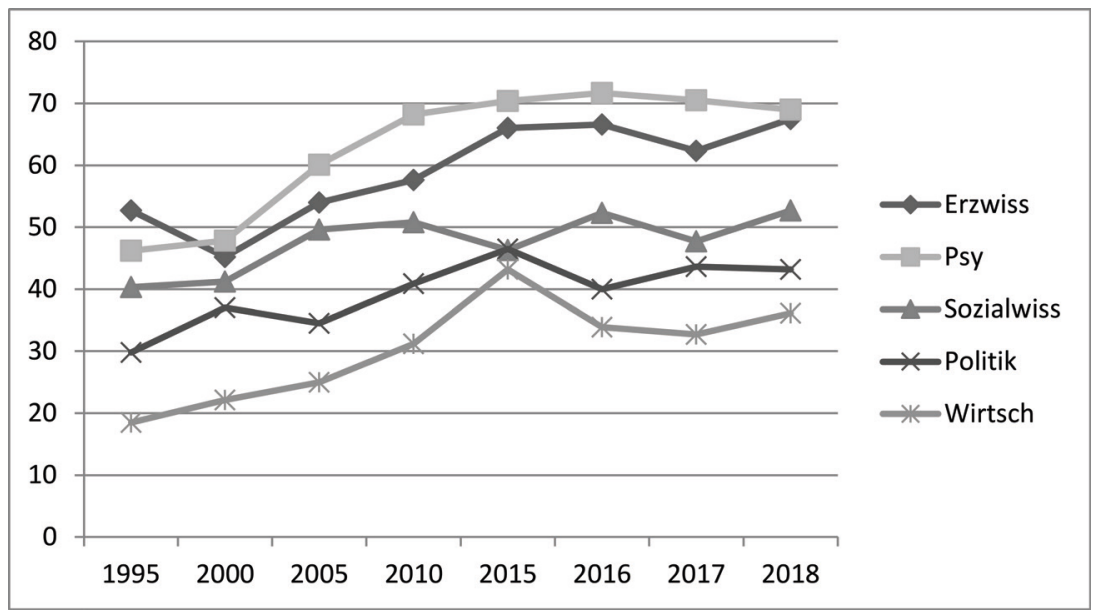

Quelle: Statistisches Bundesamt, Fachserie 11, Reihe 4.2

Der prozentuale Anteil der Frauen bei abgeschlossenen Promotionen nimmt in den einzelnen Fachgebieten unterschiedliche Entwicklungen. Während der Frauenanteil in den Politik- und Wirtschaftswissenschaften über den Zeitverlauf eine ähnliche Entwicklung zeigt, ist er in den Sozialwissenschaften durch Schwankungen charakterisiert. In der Psychologie weist er von 1995 bis 2016 eine stets steigende Tendenz auf, verharrt dann auf demselben Niveau. In der 
Erziehungswissenschaft fällt der Frauenanteil zu Ende der 1990er Jahre um 8 Prozentpunkte ab, um dann bis 2016 um 20 Prozentpunkte anzusteigen. Nach einem leichten Rückgang im Jahr 2017 erreicht er 2018 seinen Höchstwert.

\subsubsection{Habilitationen im Fächervergleich}

Die Entwicklung der Anzahl von Habilitationen seit 1995 erfolgt in den untersuchten Fachgebieten unterschiedlich. Ein Anstieg ist zunächst bei allen Disziplinen zu verzeichnen, was auch durch die Diskussion um die Einführung der Juniorprofessur und damit verbundener unsicherer Karriereplanung bedingt sein kann. Allerdings variiert der Höchstwert der Entwicklung in Zeitpunkt und Umfang zwischen den Fächern. In den Wirtschaftswissenschaften wird er bereits 2000 erreicht. Die anderen Disziplinen folgen jeweils in jährlichem Abstand bis 2004. Danach zeigt sich in allen Fachgebieten trotz einiger Schwankungen eine deutlich rückläufige Entwicklung, vgl. Tabelle 6.4. In der Psychologie sowie den Politikwissenschaften fallen die Zahlen auf das Ausgangsniveau von 1995. In den Wirtschafts- sowie den Sozial- und Erziehungswissenschaften fallen sie sogar darunter.

Tabelle 6.4: Habilitationen nach Fachgebiet 1995, 2000, 2005, 2010 und 20152018

\begin{tabular}{lccccc} 
Jahr & $\begin{array}{c}\text { Erziehungs- } \\
\text { wissenschaft }\end{array}$ & Psychologie & $\begin{array}{l}\text { Politik- } \\
\text { wissenschaft }\end{array}$ & $\begin{array}{l}\text { Sozial- } \\
\text { wissenschaft }\end{array}$ & $\begin{array}{l}\text { Wirtschafts- } \\
\text { wissenschaft }\end{array}$ \\
\hline 1995 & 36 & 41 & 15 & 35 & 51 \\
\hline 2000 & 40 & 47 & 33 & 35 & 116 \\
\hline 2005 & 49 & 56 & 27 & 38 & 85 \\
\hline 2010 & 36 & 43 & 16 & 22 & 49 \\
\hline 2015 & 26 & 42 & 11 & 21 & 54 \\
\hline 2016 & 25 & 47 & 19 & 23 & 43 \\
\hline 2017 & 26 & 33 & 15 & 21 & 49 \\
\hline 2018 & 27 & 41 & 13 & 27 & 45
\end{tabular}

1 einschließlich Sonderpädagogik und Sozialwesen

Quelle: Statistisches Bundesamt, Fachserie 11, Reihe 4.4

Der prozentuale Anteil von Frauen bei Habilitationen weist in den Politik- und Wirtschaftswissenschaften zunächst eine rückläufige Tendenz auf, erlangt dann wie in allen anderen Fachdisziplinen bis 2010 einen steigenden Verlauf, vgl. Abbildung 6.5. Die Psychologie ist das einzige Fachgebiet, in der der Frauenanteil bis 2015/2016 eine kontinuierliche Steigerung verzeichnen kann, wenngleich die Gesamtzahl an Habilitation zurückgeht. Er- 
ziehungs-, Sozial- und Politikwissenschaften weisen hingegen im Jahr 2015 einen erheblichen Rückgang auf, dem in 2016 ein mindestens ebenso großer Anstieg folgt. Nur in den Sozialwissenschaften bleibt der Anteil der Frauen dann stabil. In den Politikwissenschaften geht er seit 2016 stetig zurück und erreicht 2018 nahezu das Niveau von 1995. In der Psychologie sowie in den Erziehungs- und Wirtschaftswissenshaften geht der Frauenanteil bei Habilitationen im Jahr 2017 zurück, um dann in 2018 in ähnlichem Ausmaß wieder anzusteigen. Die Erziehungswissenschaft bekommt 2018 den größten Zuwachs.

Abbildung 6.5: Prozentualer Anteil der Frauen bei Habilitationen nach Fachgebiet 1995, 2000, 2005, 2010 und 2015-2018

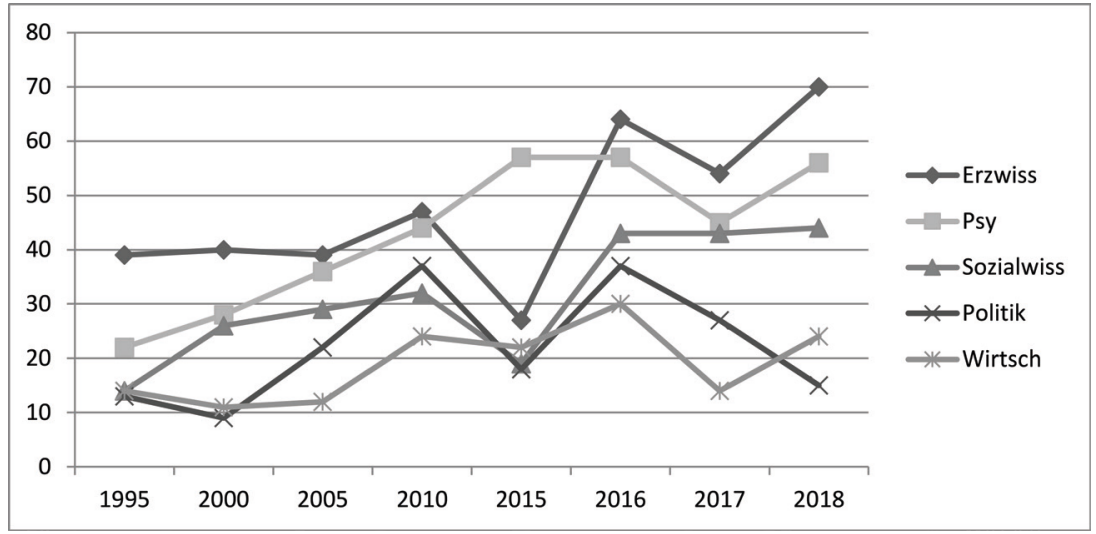

Quelle: Statistisches Bundesamt, Fachserie 11, Reihe 4.4.

\subsection{Fazit}

Die an erziehungswissenschaftlichen Fachbereichen deutscher Hochschulen abgeschlossene Zahl von Promotionen hat sich im Untersuchungszeitraum 2015-2018 im Vergleich zu 2011-2014 kaum verändert, weist aber für die letzten zwei Jahre einen nennenswerten Rückgang auf. Diese Entwicklung folgt allerdings den Zyklen vergangener Jahre. Im Untersuchungszeitraum sind an einzelnen Hochschulen die Veränderungen zu den Jahren 2011-2014 zum Teil erheblich: Während innerhalb von vier Jahren an einigen Einrichtungen ein beachtlicher Anstieg von bis zu 39 Promotionen erreicht wird, zeigen andere Hochschulen Rückgänge von bis zu 25 Arbeiten. Zur Spezifizierung wären umfangreichere Darstellungen und vertiefte Analysen erforderlich. Die unter- 
schiedliche Dynamik der Forschungsentwicklung zwischen den Hochschulstandorten wird daraus dennoch deutlich. Ein Grund für diese großen Schwankungen liegt aber auch im Auslaufen des umfangreichen Förderprogramms für den wissenschaftlichen Nachwuchs im Rahmenprogramm empirische Bildungsforschung, das erst ab 2020 eine Weiterführung erfährt.

Thematisch wird sich bei den Promotionen insbesondere der Bildungsforschung, der Sozialpädagogik, der Berufs- und Wirtschaftspädagogik sowie unterrichtsbezogenen wie auch lernspezifischen Fragestellungen zugewandt. Themen der Lehrausbildung wie auch des Lehrerberufs haben den größten Zuwachs gegenüber dem vorherigen Untersuchungszeitraum genommen. Auch die Zahl der Promotionen im frühkindlichen Bereich ist in den letzten Jahren deutlich angewachsen. Die Häufigkeit sonderpädagogischer Themen hat sich auch durch die Bearbeitung inklusiver Fragestellungen nicht wesentlich verändert. Bildungshistorisch fokussierte Promotionen nehmen deutlich ab. Die hohe Anzahl von Mehrfachzuordnungen deutet darauf hin, dass viele Themen aus einer multidisziplinären Perspektive bearbeitet werden.

Für Habilitationen ist ein deutlicher Rückgang festzustellen. Wenngleich die Zahl der Juniorprofessuren in der Erziehungswissenschaft steigt, lässt eine geringe bzw. unspezifische Datenlage noch keine gesicherten Aussagen zu diesem Karriereweg zu. Bei den Habilitationen werden überwiegend Themen aus den Forschungsfeldern Bildungsforschung, Lernen, Fachdidaktik und Bildungstheorie aufgegriffen. Aufgrund der kleinen Fallzahlen lassen sich keine Trends erkennen.

Insgesamt zeigen sich für die Erziehungswissenschaft bei der Förderung des wissenschaftlichen Nachwuchses im Vergleich zu den anderen in die Auswertung einbezogenen Fächern im Untersuchungszeitraum nur beim Frauenanteil sowie bei der Anzahl von Promotionen je 100 Professuren nennenswerte Unterschiede. Der Anteil von Frauen fällt sowohl bei den Promotionen wie auch bei den Habilitationen in der Erziehungswissenschaft deutlich höher aus als in den Politik-, Sozial- oder Wirtschaftswissenschaften. Auffällig ist der seit 2010 deutlich größere Drittmittelzuwachs in der Erziehungswissenschaft (60 \%) im Vergleich zur Psychologie (38 \%), Politik- (34\%), Sozial- (43\%) und Wirtschaftswissenschaften (26\%), der sich nicht in einer steigenden Zahl erfolgreich abgeschlossener Promotionsverfahren je Professur niederschlägt. Ein Grund dafür kann die personalintensive Einwerbung von Drittmitteln sein. Personal, das dann nicht mehr in ausreichendem Maße für die Betreuung des wissenschaftlichen Nachwuchses zur Verfügung stehen kann.

Für den Vergleich von Daten aus dem Statistischen Bundesamt mit den an die ZfPäd gemeldeten Habilitationen und Promotionen ist die grundlegend verschiedene Erhebungspraxis - Hochschulstatistikgesetz versus »Goodwill« - zu berücksichtigen. Da die Zeitschrift für Pädagogik eine der wenigen deutschen erziehungswissenschaftlichen Fachzeitschriften ist, die stark in der 
Fachcommunity wie auch internal, z.B. über den Social Science Citation Index, wahrgenommen wird, sollte eine aktuelle Darstellung der Ergebnisse aus Habilitations- und Promotionsverfahren aus den Hochschulen im Interesse aller liegen.

\section{Literatur}

DGfE (= Deutsche Gesellschaft für Erziehungswissenschaft) (2011): Publikationsbasierte Dissertationen in der Erziehungswissenschaft - eine Stellungnahme. http://www. dgfe.de/fileadmin/OrdnerRedakteure/Stellungnahmen/2011_Publikationsbasierte_Promotionen.pdf [Zugriff: 31.10.2019]

Kauder, Peter (2012): Die Problematik der der "Zeitschrift für Pädagogik « gemeldeten Promotionen und Habilitationen. In: Zeitschrift für Pädagogik 58, 5, S. 734-756.

Martini, Renate/Schmidt-Hertha, Bernhard (2012): Förderung des wissenschaftlichen Nachwuchses. In:DGfE:Datenreport Erziehungswissenschaft2012.Hrsg.v. Thole, W./ Faulstich-Wieland, H./Horn, K.-P./Weishaupt, H./Züchner, I. Schriften der Deutschen Gesellschaft für Erziehungswissenschaft. Opladen u.a.: Verlag Barbara Budrich, S. 181-192.

Martini, Renate/Dees, Werner (2012): Qualifizierungsarbeiten in der Bildungsforschung 1998-2007. In: Weishaupt, H./Rittberger, M. (Hrsg.): Bildungsforschung in Deutschland - eine Situationsanalyse. Bildungsforschung, Bd. 37, S. 43-64 http://www.pedocs. de/volltexte/2013/8206/pdf/Martini_Dees_2012_Qualifizierungsschriften_in_der_ Bildungsforschung_1998_bis_2007.pdf [Zugriff: 31.10.2019]

Martini, Renate (2016): Förderung des wissenschaftlichen Nachwuchses. In: DGfE: Datenreport Erziehungswissenschaft 2016. Hrsg. v. Koller, H.-C./Faulstich-Wieland, H./ Weishaupt, H./Züchner, I. Schriften der Deutschen Gesellschaft für Erziehungswissenschaft. Opladen u.a.: Verlag Barbara Budrich, S. 179-190. https://www.pedocs.de/ frontdoor.php?source_opus=12228 [Zugriff: 31.10.2019]

Preißler, Ulrike (2019): Die Besoldung der Juniorprofessur: W 1 im Überblick beim Bund und den Ländern. In: Forschung und Lehre 26, S. 538-540.

Vollmar, Meike (2019): Neue Promovierendenstatistik: Analyse der ersten Erhebung 2017. In: Wirtschaft und Statistik 1, S. 68-80. 